\title{
Weighted Norm Inequalities for the Restriction of Fourier Transform to $S^{n-1}$
}

\section{LOU HONGWEI \\ Ninbo University}

In $[1,3,5]$, E. M. Stein, C. Fefferman, A. Zygmund, and P. Tomas considered the restriction of Fouier transform to the unit sphere $S^{n-1}$. They proved that the a-priori inequality

$$
\left(\int_{S^{n-1}}|\hat{f}(\theta)|^{q} d \theta\right)^{1 / q} \leq C\|f\|_{p} \quad f \in S\left(\mathbb{R}^{n}\right)
$$

holds if and only if $1 \leq p<4 / 3, q \leq p^{\prime} / 3$ when $n=2$. When $n \geq 3$, they proved that (1) holds if $1 \leq p \leq \frac{2(n+1)}{n+3}$ and $q \leq \frac{n-1}{n+1} p^{\prime}$.

Now we consider the weighted norm inequalities for the restriction of Fourier transform to $S^{n-1}$ :

$$
\left(\int_{S^{n-1}}|\hat{f}(\theta)|^{q} d \theta\right)^{1 / q} \leq C\left(\int_{\mathbb{R}^{n}}|f(x)|^{p} \varphi(|x|) d x\right)^{1 / p} \quad f \in S
$$

where $1 \leq q<\infty, 1 \leq p \leq 2$ and $\varphi(r) \geq 0$ is a measurable function on $[0, \infty)$, we prove that:

Theorem 1: Let $p=2,1 \leq q \leq 2, N=\{0,1,2,3, \cdots\}$, if:

$$
\sup _{k \in \mathcal{N}} \int_{0}^{\infty} \frac{r}{\varphi(r)} J_{k+\frac{2-2}{2}}^{2}(2 \pi r) d r<\infty
$$

then (2) holds. When $n=2$ or $q=2,(2)$ holds if and only if (3) holds.

Theorem 2: Let $\varphi(r)=r^{a}, 1 \leq q \leq 2 \leq p<\infty$, then (2) holds if and only if $\frac{n+1}{2} p-n<a<n(p-1)$.

On the other hand, we have:

Theorem 3: Let $g(\theta) \in L^{2}\left(S^{n-1}\right), q \leq \frac{2 n}{n-1}$, if $\|(g d \theta)(x)\|_{q}<\infty$ then $g(\theta)=0$ for almost every $\theta \in S^{n-1}$, where we define $(g d \theta)(x)=\int_{S^{n-1}} g(\theta) e^{-2 \pi i x \cdot \theta} d \theta$.

Acknowledgment: I am grateful to my advisers, Prof. Wan Si-Lei and Prof. Shi Xian-Liang, for their many helpful suggestions and discussions.

\section{References}

1. C. Fefferman, Acta Math. 124(1970), 9-36.

2. E. M. Stein \& G. Weiss, Introduction to Fourier analysis in Euclidean spaces, Princeton University Press. Princeton, NJ.1975.

3. P. Tomas, Bull. Am. Math. Soc. 81(1975), 477-478.

4. G. N. Watson, Theory of Bessel functions, Cambridge University Press, Cambridge, 1944.

5. A. Zygmund, Studia Math. 50(1974), 189-201. 